\title{
Expression of p16 and SATB1 in Invasive Ductal Breast Cancer - A Preliminary Study
}

\author{
CHRISTOPHER KOBIERZYCKI ${ }^{1}$, JEDRZEJ GRZEGRZOLKA ${ }^{1}$, \\ NATALIA GLATZEL-PLUCINSKA ${ }^{1}$, ALEKSANDRA PIOTROWSKA ${ }^{1}$, ANDRZEJ WOJNAR ${ }^{2}$, \\ BEATA SMOLARZ ${ }^{3}$, HANNA ROMANOWICZ ${ }^{3}$ and PIOTR DZIEGIEL ${ }^{1,4}$ \\ ${ }^{1}$ Division of Histology and Embryology, Department of Human Morphology and Embryology, \\ Wroclaw Medical University, Wroclaw, Poland; \\ ${ }^{2}$ Department of Pathomorphology, Lower Silesian Oncology Centre, Wroclaw, Poland; \\ ${ }^{3}$ Department of Pathology, Polish Mother's Memorial Hospital Research Institute, Lodz, Poland; \\ ${ }^{4}$ Department of Physiotherapy, University School of Physical Education, Wroclaw, Poland
}

\begin{abstract}
Background/Aim: An impaired cell-cycle control and genetic material organization are crucial elements of carcinogenesis. pl6 is a tumor suppressor protein which decelerates promotion of the cells from $G_{1}$ to $S$ phase, whereas special AT-rich sequence-binding protein 1 (SATB1) is a nuclear matrix protein that binds to specific regions of the DNA and ensures its proper organization and function. Increased levels of both markers are observed in various types of cancers. The aim of this study was to investigate the expression of p16 and SATB1 proteins in regard to expression of the Ki-67 antigen and available clinicopathological data (i.a. receptor status, staging and grading). Materials and Methods: The study was performed on 130 samples of archived invasive ductal breast cancers. Immunohistochemical reactions were performed on freshly prepared tissue microarrays and subsequently scanned by a histologic scanner. Reactions were evaluated separately in the cytoplasm (p16c, SATB1c) and nucleus (p16n, SATB1n, Ki-67) with use of a quantification software under researcher supervision. Results: Expression was observed for $\mathrm{Ki}-67$ in 100\%, p16c in 90\%, p16n in 89.2\%, SATB1c in $98.5 \%$ and SATB1n in $87.7 \%$ of cancer cases. Statistical analysis showed strong positive correlations: p16c vs.p16n and SATB1c vs. SATBIn ( $p<0.001$ for both) and weak
\end{abstract}

This article is freely accessible online.

Correspondence to: Christopher Kobierzycki, MD, Ph.D., Division of Histology and Embryology, Department of Human Morphology and Embryology, Wroclaw Medical University, Chalubinskiego 6a, 50-368 Wroclaw, Poland. Tel: +48 717841682, Fax: +48 717840082, e-mail: christopher.kobierzycki@umed.wroc.pl

Key Words: p16, SATB1, Ki-67, TMA, tissue microarray, proliferation, breast cancer. positive correlations: $p 16 c$ vs. SATB1c and p16c vs. SATBIn ( $p=0.008, p=0.027 ;$ respectively). Expression of $p 16 n$ was stronger in $G_{1}$ vs. $G_{2}(p=0.034)$ while Ki-67 expression was stronger in cases with negative progesterone receptor status $(p=0.011)$. All other analyzed associations were statistically insignificant. Conclusion: A weak association between immunohistochemical expression of p16 and SATB1 indicated limited possibility of their independent usage. Further studies concerning determination of a wider panel of proteins controlling cell cycle should be considered.

Malignant transformation of the cell is a complex process which originates from pathological gene expression. In many hypotheses, abnormalities associated with cell cycle regulation have been pointed. Transformation may be the result of the impaired function of two controlling points: p53 and retinoblastoma protein (mediated by p16). Simultaneously, structural alterations in chromatin organization can be observed. They may be considered as cell cycle dependent or independent events, resulting in gene expression dysregulation, i.a. by specific transcription factors involvement $(1,2)$.

p16 protein plays an important role in regulation of the cell cycle. It acts as a tumor suppressor by binding to cyclindependent kinases 4/6 and prevents interaction of cyclin D1 with retinoblastoma $(\mathrm{Rb})$ protein. It ultimately inhibits the downstream activities of transcription factors, such as E2F1, and finally arrests cell proliferation. Hypermethylation, mutation, or deletion of p16 leads to downregulation of the gene which may increase the incidence of some malignancies, i.a. melanoma, oropharyngeal squamous cell carcinoma, cervical cancer, and esophageal cancer $(3,4)$. Currently, in gynecological screening tests, evaluation of p16 is performed to improve the histological diagnostic accuracy of cervical intraepithelial neoplasia grade 3 . It is available as a commercial kit for combined evaluation of p16 and Ki-67 (CINtec; Ventana, Tucson, AZ, USA) (5). 
Special AT-rich sequence binding protein 1 (SATB1) is a genome organizer protein that facilitates various intracellular processes. It provides a nuclear architectural platform that binds hundreds of genes, through its interaction with specific genomic sequences. It allows parallel regulation of many genes expression enabling thereby cells to alter their function (6). SATB1 as a matrix attachment region binding protein regulates the genes by folding chromatin into loop domain (7). It was found to regulate gene expression in thymocytes and pre-B-cells but recent studies have shown that SATB1 promotes tumor growth and metastasis through chromatin gene recombination in many neoplasms such as breast, gastrointestinal tract (predominantly colorectal region), liver, laryngeal, lung, thyroid, urinary bladder, ovarian and prostate cancers, melanomas, osteosarcomas, gliomas or some leukemias and lymphomas (826). There is evidence that in some cancers, e.g. colorectal, depletion of SATB1 expression is associated with poor prognosis $(12,27)$ whereas in non-small cell lung cancer strong expression of SATB1 correlates with a better overall survival of patients (15). Moreover, in breast cancer it is suggested that combined evaluation of Ki67 and SATB1 as a ratio may be an independent prognostic factor of overall survival (28).

In order to understand the processes leading to malignant transformation in breast cancer, the expression of p16 and SATB1 protein in regard to expression of routinely performed proliferative marker Ki-67 and available clinicopathological data (i.a. receptor status, staging and grading) was investigated.

\section{Materials and Methods}

Patients. The material for the study consisted of 130 archived paraffin embedded samples of invasive ductal breast cancers. The patients aged 26-81 years old (median 57 years old) were of female sex. Relevant available clinicopathological data, i.e. receptor status, staging and grading are presented in Table I.

Tissue microarray (TMA) construction. Hematoxylin and eosin stained (HE) 6- $\mu \mathrm{m}$ thick paraffin sections were prepared to verify histopathological diagnosis and evaluate sample usefulness for further analyses. In brief, slides were scanned utilizing histologic scanner Pannoramic MIDI (3DHistech, Budapest, Hungary). Subsequently, scans were examined by two independent pathologists and areas of interest with potentially the highest tumor cell content from non-necrotic areas were marked electronically. Afterwards, for TMA construction, from the corresponding paraffin donor blocks, triplicate tissue core punches $(2 \mathrm{~mm})$ for every case were obtained (TMA Grand Master; 3DHistech).

Immunohistochemistry (IHC). Immunohistochemical reactions were performed on 4- $\mu \mathrm{m}$ paraffin sections obtained from TMA blocks mounted on Superfrost Plus slides (Menzel Gläser, Braunschweig, Germany). The sections were dewaxed, re-hydrated and the epitopes were exposed using Pre-Treatment Link Rinse Station and Target Retrieval Solution ( $\mathrm{pH} 6$ for Ki-67; pH 9 for p16, SATB1; 97 ${ }^{\circ} \mathrm{C}, 20$ min) (Dako, Glostrup, Denmark). Activity of endogenous peroxidase was blocked by 5 min exposure to Peroxidase-Blocking Reagent (Dako). The sections were then rinsed with Wash Buffer and incubated
Table I. Clinicopathological features of patients with invasive ductal breast cancer.

\begin{tabular}{lcr}
\hline Feature & $\mathrm{N}$ & $\%$ \\
\hline Grade & & \\
G1 & 12 & 9.23 \\
G2 & 77 & 59.23 \\
G3 & 41 & 31.54 \\
TNM & & \\
pT1-2 & 125 & 96.15 \\
pT3-4 & 5 & 3.85 \\
pN0 & 77 & 59.23 \\
pN1-3 & 53 & 40.77 \\
Stage & & \\
I-IIB & 120 & 92.30 \\
IIIA-IV & 10 & 7.70 \\
Receptor status & & \\
ER (+) & $83 / 130$ & 63.85 \\
PgR (+) & $76 / 130$ & 58.46 \\
HER-2 (+) & $13 / 70$ & 18.58 \\
\hline
\end{tabular}

for $20 \mathrm{~min}$ at room temperature with the following primary antibodies against Ki-67 (MIB-1; Ready-to-use; Dako), p16 (G175-405; 1:100+linker; BP Pharmingen, CA, USA), and SATB1 (EPR3951; 1:100; GeneTex, Hsinchu, Taiwan). Secondary goat anti-mouse and anti-rabbit antibodies coupled to a dextran core, linked to horseradish peroxidase, were applied and subsequent visualization was performed using the EnVision ${ }^{\mathrm{TM}}$ FLEX+ system (Dako) according to the manufacturer's instructions. All IHC reactions were performed in an automated staining platform, Autostainer Link48 (Dako). The reactions were visualized using 3,3'-diaminobenzidine tetrachlorohydrate (DAB+ chromogen). All slides were counterstained with Mayer's hematoxylin.

Evaluation of IHC reaction. The slides were scanned utilizing histologic scanner Pannoramic MIDI (3DHistech). Reactions were evaluated separately in cytoplasm (p16c, SATB1c) and nucleus (p16n, SATB1n, Ki-67) with usage of Quant Center Software (3DHistech) under researcher supervision. For every case three TMA cores were quantified by the algorithm SCORE (range=0-8) and the final result was an average count.

Statistical analysis. The results were subjected to statistical analysis using the Prism 5.0 software (GraphPad, La Jolla, CA, USA) utilizing Kolmogorov-Smirnov, Spearman's rank correlation, Kruskal-Wallis, Mann-Whitney and Wilcoxon tests. Kaplan-Maier curves were performed. In all analyses, results were considered to be statistically significant for $p<0.05$.

\section{Results}

Expression was observed for Ki-67 in $100 \%$, p16c in $90 \%$, p16n in $89,2 \%$, SATB1c in $98.5 \%$ and SATB $1 \mathrm{n}$ in $87.7 \%$ of cancer cases. Analysis of Ki-67, p16 and SATB 1 expression was conducted on TMA serial sections (Figure 1A-F). Statistical analysis showed strong positive correlations: $\mathrm{p} 16 \mathrm{c}$ vs. $\mathrm{p} 16 \mathrm{n}$ and SATB1c vs. SATB1n $(\mathrm{r}=0.715, \mathrm{r}=0.759$; 


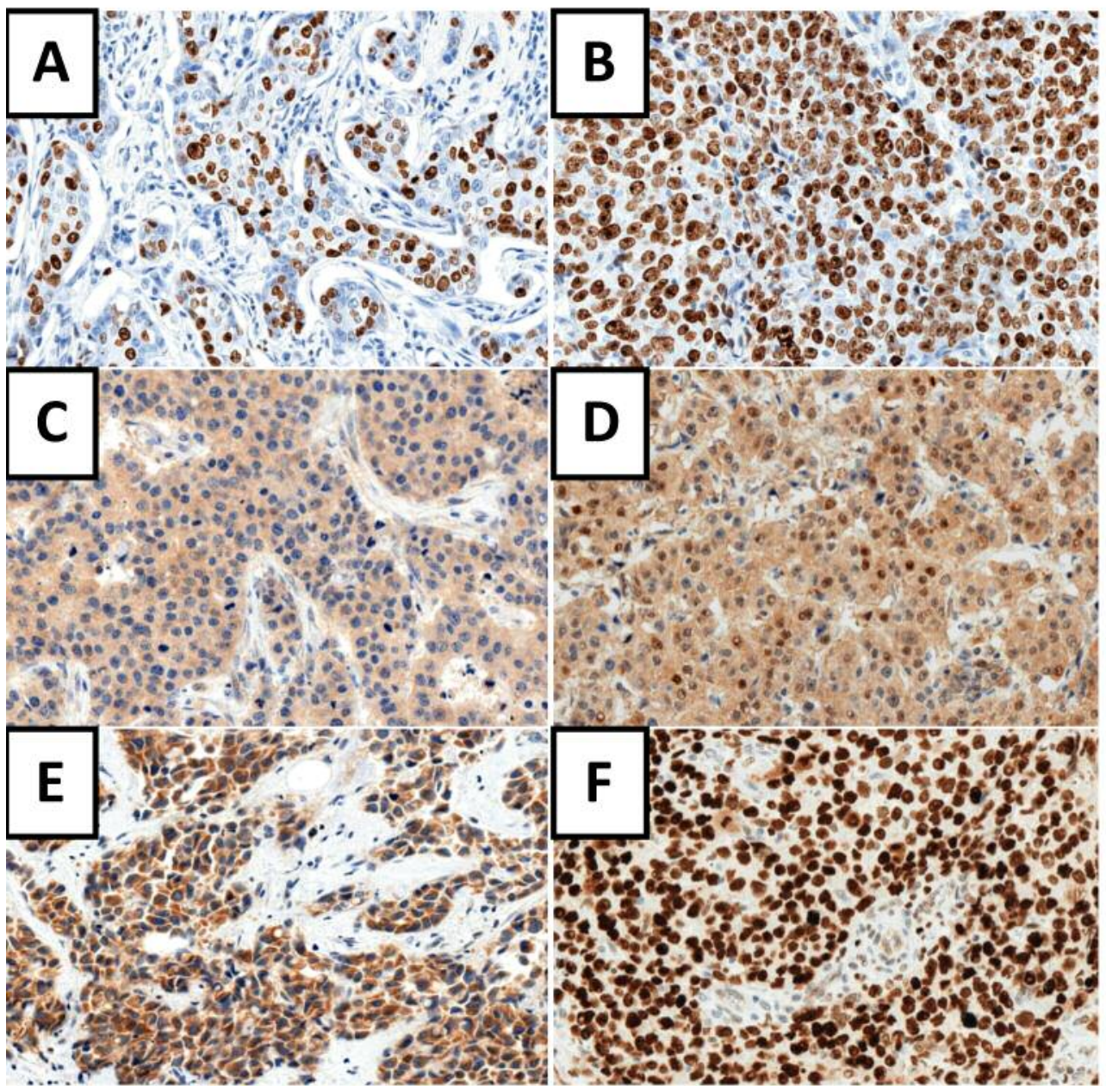

Figure 1. Immunohistochemical expression of Ki-67 (weak, A; strong, B), p16c (cytoplasmic, C), p16n (nuclear, D), SATB1c (cytoplasmic, E), and (nuclear, F). Magnification $\times 200$.

respectively and $p<0.001$ for both) and weak positive correlation: $\mathrm{p} 16 \mathrm{c} v s$. SATB1c $(\mathrm{r}=0.220, p=0.008)$ and $\mathrm{p} 16 \mathrm{c}$ vs. SATB1n ( $\mathrm{r}=0.185, p=0.027)$ (Spearman's rank correlation test, Table II). Expression of p16n was stronger in $\mathrm{G}_{1} v s . \mathrm{G}_{2}$ $(p=0.034)$ and $\mathrm{Ki}-67$ expression was stronger in cases with negative progesterone receptor status $(p=0.011$, MannWhitney test). No association between expression of studied markers and staging or TNM scale was found. Moreover, separate and combined survival analyses in regard to p16c, p16n, SATB1c and SATB1n expression disclosed statistically insignificant results (Figure 2).

\section{Discussion}

The p16 tumor suppressor gene $(C D K N 2 A)$ located on chromosome 9 p21 is a member of the INK4 class of cell cycle inhibitors. The p16 protein binds to cyclin-dependent kinases 4/6 what prevents formation of active complex with
Table II. Spearman correlation test results.

\begin{tabular}{|c|c|c|c|c|c|}
\hline & $\mathrm{Ki}-67$ & p16n & $\mathrm{p} 16 \mathrm{c}$ & SATB1n & SATB1c \\
\hline $\begin{array}{l}\text { Ki-67 } \\
\text { p16n }\end{array}$ & & NS & $\begin{array}{c}\mathrm{NS} \\
\mathrm{r}=0.715 \\
p<0.001\end{array}$ & $\begin{array}{l}\text { NS } \\
\text { NS }\end{array}$ & $\begin{array}{l}\text { NS } \\
\text { NS }\end{array}$ \\
\hline $\mathrm{p} 16 \mathrm{c}$ & & & & $\begin{array}{l}\mathrm{r}=0.185 \\
p=0.027\end{array}$ & $\begin{array}{l}\mathrm{r}=0.220 \\
p=0.008\end{array}$ \\
\hline SATB1n & & & & & $\begin{array}{l}\mathrm{r}=0.759 \\
p<0.001\end{array}$ \\
\hline
\end{tabular}

cyclin D1. This blocks phosphorylation of $\mathrm{Rb}$ protein hindering dissociation of E2F transcription factor and finally stopping transition from $\mathrm{G} 1$ to $\mathrm{S}$ phase $(29,30)$. Expression of p16 protein is increased in aging cells inducing apoptosis 


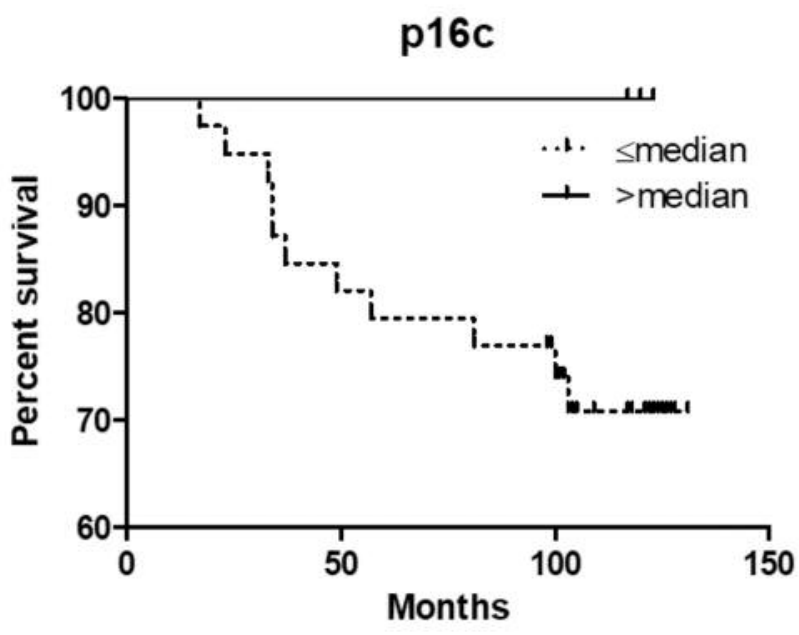

SATB1C

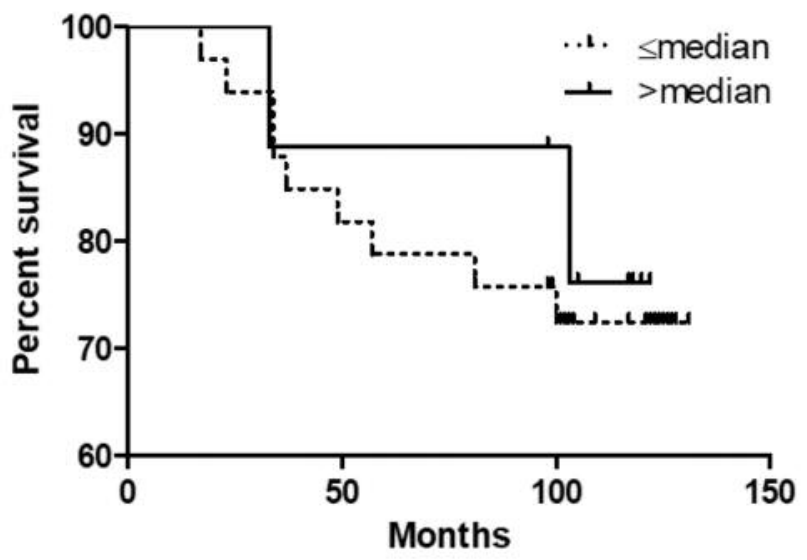

Figure 2. Kaplan-Meier curves for studied markers - p16 and SATB1.

process. Similar mechanism was disclosed in metaplastic and atrophic cells $(30,31)$. There is evidence that in non-HPVrelated malignant neoplasms, i.e. breast, pancreas, colon tumors as well head and neck carcinomas are related to smoking, p16 function is lost by gene deletions, mutations, or epigenetic silencing. This results in negative IHC findings in the abovementioned cancers $(3,29-31)$. On the other hand, IHC expression of p16 is routinely performed in gynecological diagnostics, mostly in cervical dysplasia screening, combined with Ki-67 evaluation using commercial kits, e.g. CINtec (4). The disruption of the p16cyclin D1-CDK4/6-Rb pathway is pointed in many human cancers, however its significance in breast carcinogenesis is still controversial. In the present study correlation between $\mathrm{p} 16 \mathrm{c}$ and $\mathrm{p} 16 \mathrm{n}$ was strong $(\mathrm{r}=0.715, p<0.001)$. However, no association with other studied markers, i.e. Ki-67 and SATB1 was found. Expression of p16n was stronger in $\mathrm{G}_{1}$ vs. $\mathrm{G}_{2}$
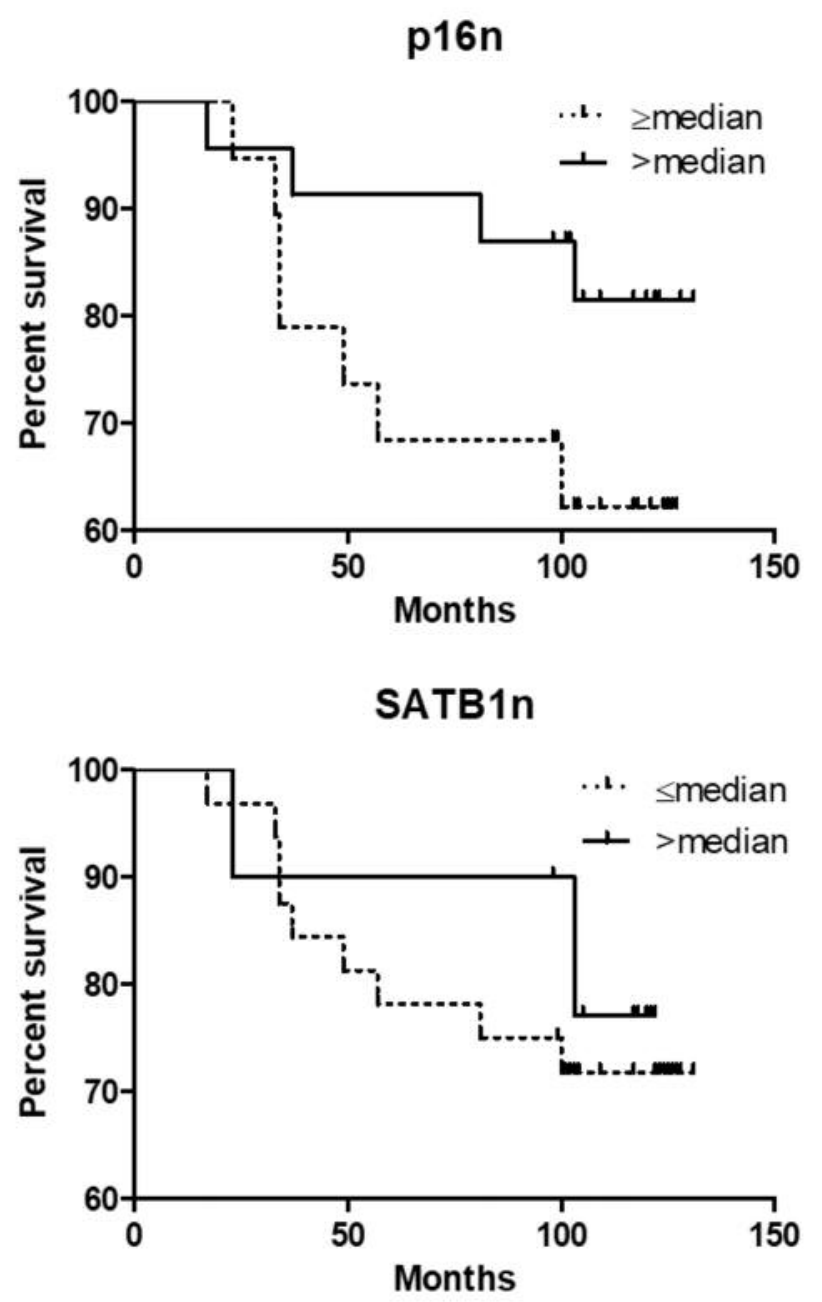

$(p=0.034)$. It indirectly stays in line with observation by Peurala et al. who postulated that p16 expression correlates with a better prognosis and may function independently (without CDK4) in human breast cancer (32). However, there is also evidence that $\mathrm{p} 16$ promotes growth and mobility potential of breast cancer by activation IL-6/JAK2/STAT3 pathway (33). We performed also separate survival analysis for $\mathrm{p} 16 \mathrm{c}$ and $\mathrm{p} 16 \mathrm{n}$ expression as well in combination with Ki-67 expression. Results were statistically insignificant. There are some speculations about potential of 9p21 locus as a therapeutic target and prognostic marker in breast cancer, i.a. due to hypermethylation of the p16 gene in malignancies (34). Currently, studies are ongoing.

SATB1 (special AT-rich sequence-binding protein-1) located on chromosome $3 \mathrm{p} 24$ is a global chromatin organizer and transcription factor, mostly responsible for higher-order chromatin architecture and gene regulation (35, 
36). Aberrant expression of SATB 1 has been shown to promote growth and metastasis of various neoplasms (8-26). The expression of SATB1 simultaneously increases with the progression of cancers and it dynamically reprograms the expression of genes that are involved in carcinogenesis (36). It determines specific epigenetic modifications at target gene loci, directly up-regulating metastasis-associated genes while down-regulating tumor-suppressor genes. As SATB1 reprogrammes chromatin organization and the transcription profiles to promote growth and metastasis in breast cancer, it is presumed that mutation of $S A T B 1$ gene might affect not only cell cycle progression but also apoptosis pathway in breast cancer (37). In this study, the association between SATB1 protein expression with routinely performed marker of proliferation - Ki-67 antigen as well one of very important controller of cell cycle - p16 was investigated. Similarly to p16, SATB1 expression was analyzed in cytoplasm and nucleus separately. Except of the strong correlation between SATB1c and SATB1n no statistically significant associations were found. This stays in line with results of our previous study (38) as well as of the study by Laurinavicius et al. (39). They analyzed a set of 10 IHC markers - ER, PR, HER2, Ki-67, AR, BCL2, HIF-1 $\alpha$, SATB1, p53, and p16 in invasive ductal breast cancer. They observed insignificant associations between SATB1 and p16 in regard to other tested markers, except HIF-1 $\alpha$. They speculated that SATB1 and HIF- $1 \alpha$ may be important markers of estrogen-positive cancers, whereas their biological and clinical significance remains to be elucidated (39). In our previous study we analyzed expression of SATB1 and $\mathrm{Ki}-67$ in regard to receptor status. We found moderate correlation between mentioned markers ( $\mathrm{r}=0.392$, $p=0.032$ ) only in estrogen-negative tumors (38). Nevertheless, in the present study no associations with receptor status and the studied markers were disclosed. Moreover, Laurinavicius et al. suggested that in breast cancer combined evaluation of Ki67/SATB1 ratio may be an independent prognostic factor of overall survival (28). We also performed separate survival analysis for SATB1c and SATB1n expression as well in combination with Ki-67 expression. Results were statistically insignificant.

\section{Conclusion}

Both studied proteins are present in diagnostic pathology. Their use in screening, differential diagnostics and prognostic value in breast cancer is still unknown. A weak association between immunohistochemical expression of p16 and SATB1 indicates limited possibility of their independent usage. In regard to received results and literature data, further studies concerning the determination of a wider panel of proteins controlling cell cycle should be considered, probably in combination.

\section{Acknowledgements}

This research was financially supported by the Ministry of Science and Higher Education with a grant for Young Scientists no. Pbmn142.

\section{References}

1 Mirkovitch J, Mirault ME and Laemmli UK: Organization of the higher-order chromatin loop: specific DNA attachment sites on nuclear scaffold. Cell 39: 223-232, 1984.

2 Nelson WG, Pienta KJ, Barrack ER and Coffey DS: The role of the nuclear matrix in the organization and function of DNA. Annu Rev Biophys Biophys Chem 15: 457-475, 1986.

3 Mahajan A: Practical issues in the application of p16 immunohistochemistry in diagnostic pathology. Hum Pathol 51: 64-74, 2016.

4 Ciesielska U, Zatonski T, Nowinska K, Ratajczak-Wielgomas K, Grzegrzolka J, Piotrowska A, Olbromski M, Pula B, PodhorskaOkolow M and Dziegiel P: Expression of cell cycle-related proteins p16, p27 and Ki-67 proliferating marker in laryngeal squamous cell carcinomas and in laryngeal papillomas. Anticancer Res 37: 2407-2415, 2017.

5 Tjalma WAA: Diagnostic performance of dual-staining cytology for cervical cancer screening: A systematic literature review. Eur J Obstet Gynecol Reprod Biol 210: 275-280, 2017.

6 Yasui D, Miyano M, Cai S, Varga-Weisz P and KohwiShigematsu T: SATB1 targets chromatin remodelling to regulate genes over long distances. Nature 10: 641-645, 2002.

7 Galande S, Purbey PK, Notani D and Kumar PP: The third dimension of gene regulation: organization of dynamic chromatin loopscape by SATB1. Curr Opin Genet Dev 17: 408-414, 2007.

8 Kohwi-Shigematsu T, Poterlowicz K, Ordinario E, Han HJ, Botchkarev VA and Kohwi Y: Genome organizing function of SATB1 in tumor progression. Semin Cancer Biol 23: 72-79, 2013.

9 Cai S, Han HJ and Kohwi-Shigematsu T: Tissue-specific nuclear architecture and gene expression regulated by SATB1. Nat Genet 34: 42-51, 2003.

10 Zheng J: Is SATB1 a master regulator in breast cancer growth and metastasis? Womens Health (Lond Engl) 4: 329-332, 2008.

11 Cheng C, Lu X, Wang G, Zheng L, Shu X, Zhu S, Liu K, Wu K and Tong Q: Expression of SATB 1 and heparanase in gastric cancer and its relationship to clinicopathologic features. APMIS 118: 855-863, 2010.

12 Fang XF, Hou ZB, Dai XZ, Chen C, Ge J, Shen H, Li XF, Yu LK and Yuan Y: Special AT-rich sequence-binding protein 1 promotes cell growth and metastasis in colorectal cancer. World J Gastroenterol 19: 2331-2339, 2013.

13 Kapoor S: SATB1 expression and its association with tumour prognosis in systemic malignancies: an evolving concept in oncology. Liver Int 33: 322, 2013.

14 Zhao XD, Ji WY, Zhang W, He LX, Yang J, Liang HJ and Wang LL: Overexpression of SATB1 in laryngeal squamous cell carcinoma. ORL J Otorhinolaryngol Relat Spec 72: 1-5, 2010.

15 Glatzel-Plucinska N, Piotrowska A, Grzegrzolka J, Olbromski M, Rzechonek A, Dziegiel P and Podhorska-Okolow M: SATB1 level correlates with $\mathrm{Ki}-67$ expression and is a positive prognostic factor in non-small cell lung carcinoma. Anticancer Res 38: 723-736, 2018. 
16 Huang B, Zhou H, Wang X and Liu Z: Silencing SATB1 with siRNA inhibits the proliferation and invasion of small cell lung cancer cells. Cancer Cell Int 13: 8, 2013.

17 Murono S, Tsuji A, Endo K, Kondo S, Wakisaka N and Yoshizaki T: Immunohistochemical detection of SATB1 is independent of thyroid cancer differentiation. Laryngoscope 123: 2909-2912, 2013

18 Han B, Luan L, Xu Z and Wu B: Expression and biological roles of SATB1 in human bladder cancer. Tumour Biol 34: 2943-2949, 2013.

19 Nodin B, Hedner C, Uhlén M and Jirström K: Expression of the global regulator SATB1 is an independent factor of poor prognosis in high grade epithelial ovarian cancer. J Ovarian Res 5: 24,2012

20 Xiang J, Zhou L, Li S, Xi X, Zhang J, Wang Y, Yang Y, Liu X and Wan X: AT-rich sequence binding protein 1: Contribution to tumor progression and metastasis of human ovarian carcinoma. Oncol Lett 4: 865-870, 2012.

21 Mao L, Yang C, Wang J, Li W, Wen R, Chen J and Zheng J: SATB1 is overexpressed in metastatic prostate cancer and promotes prostate cancer cell growth and invasion. J Transl Med 11: 111, 2013

22 Chen H, Takahara M, Oba J, Xie L, Chiba T, Takeuchi S, Tu Y, Nakahara T, Uchi H, Moroi Y and Furue M: Clinicopathologic and prognostic significance of SATB1 in cutaneous malignant melanoma. J Dermatol Sci 64: 39-44, 2011.

23 Zhang H, Qu S, Li S, Wang Y, Li Y, Wang Y, Wang Z and Li R: Silencing SATB1 inhibits proliferation of human osteosarcoma U2OS cells. Mol Cell Biochem 378: 39-45, 2013.

24 Chu SH, Ma YB, Feng DF, Zhang H, Zhu ZA, Li ZQ and Jiang PC: Upregulation of SATB1 is associated with the development and progression of glioma. J Transl Med 10: 149, 2012.

25 Han S, Xia J, Qin X, Han S and Wu A: Phosphorylated SATB1 is associated with the progression and prognosis of glioma. Cell Death Dis 4: e901, 2013.

26 Jankowska-Konsur A, Kobierzycki Ch, Reich A, Grzegrzolka J, Bieniek A and Dziegiel P: Expression of SATB1, MTI/II and Ki67 in mycosis fungoides. Anticancer Res 36: 189-197, 2016.

27 Nodin B, Johannesson H, Wangefjord S, O'Connor DP, Lindquist KE, Uhlén M, Jirström K and Eberhard J: Molecular correlates and prognostic significance of SATB1 expression in colorectal cancer. Diagn Pathol 7: 115, 2012.

28 Laurinavicius A, Green AR, Laurinaviciene A, Smailyte G, Ostapenko V, Meskauskas R and Ellis IO: Ki67/SATB1 ratio is an independent prognostic factor of overall survival in patients with early hormone receptor-positive invasive ductal breast carcinoma. Oncotarget 6: 41134-41145, 2015.
29 Serrano M: The tumor suppressor protein p16INK4a. Exp Cell Res 23: 7-13, 1997.

$30 \mathrm{Kim}$ WY and Sharpless NE: The regulation of INK4/ARF in cancer and aging. Cell 127: 265-275, 2006.

31 Kotake Y, Naemura M, Murasaki C, Inoue Y and Okamoto H: Transcriptional regulation of the p16 tumor suppressor gene. Anticancer Res 35: 4397-4401, 2015.

32 Peurala E, Koivunen P, Haapasaari KM, Bloigu R and JukkolaVuorinen A: The prognostic significance and value of cyclin D1, CDK4 and p16 in human breast cancer. Breast Cancer Res 15: R5, 2013

33 Wang L, Zhan X, Shen X, Li M, Yang J, Yu W, Chen H, Jin B and Mao Z: P16 promotes the growth and mobility potential of breast cancer both in vitro and in vivo: the key role of the activation of IL-6/JAK2/STAT3 signaling. Mol Cell Biochem, 2018. doi: 10.1007/s11010-018-3281-4. [Epub ahead of print]

34 Rivandi M, Khorrami MS, Fiuji H, Shahidsales S, Hasanzadeh M, Jazayeri MH, Hassanian SM, Ferns GA, Saghafi N and Avan A: The 9p21 locus: A potential therapeutic target and prognostic marker in breast cancer. J Cell Physiol 233: 5170-5179, 2018.

35 Pavan Kumar P, Purbey PK, Sinha CK, Notani D, Limaye A, Jayani RS and Galande S: Phosphorylation of SATB1, a global gene regulator, acts as a molecular switch regulating its transcriptional activity in vivo. Mol Cell 22: 231-243, 2006.

36 Cai S, Lee CC and Kohwi-Shigematsu T: SATB1 packages densely looped, transcriptionally active chromatin for coordinated expression of cytokine genes. Nat Genet 38: 1278-1288, 2006.

37 Mir R, Pradhan SJ and Galande S: Chromatin organizer SATB1 as a novel molecular target for cancer therapy. Curr Drug Targets 13: 1603-1615, 2012.

38 Kobierzycki C, Wojnar A and Dziegiel P: Expression of SATB1 protein in the ductal breast carcinoma tissue microarrays preliminary study. Folia Histochem Cytobiol 51: 333-338, 2013.

39 Laurinavicius A, Laurinaviciene A, Ostapenko V, Dasevicius D, Jarmalaite $S$ and Lazutka J: Immunohistochemistry profiles of breast ductal carcinoma: factor analysis of digital image analysis data. Diagn Pathol 7: 27, 2012 